\title{
Diferencias morfológicas de material reproductivo de marango acriollado (Moringa oleifera Lam.) y variedad mejorada (Moringa oleifera var. PKM)
}

\section{Morphological differences of reproductive material of native marango (Moringa oleifera Lam.) and improved variety (Moringa oleifera var. PKM)}

\author{
Álvaro Noguera-Talavera1, Nadir Reyes-Sánchez ${ }^{1}$, Bryan Mendieta-Araica1, Camilo Gutiérrez ${ }^{2}$, Diego Lino Macario ${ }^{3}$ \\ ${ }^{1}$ Docentes investigadores de la Universidad Nacional Agraria, \\ ${ }^{2}$ Investigador Instituto Nicaragüense de Tecnología Agropecuaria (INTA) \\ ${ }^{3}$ Egresado Ingeniería Forestal
}

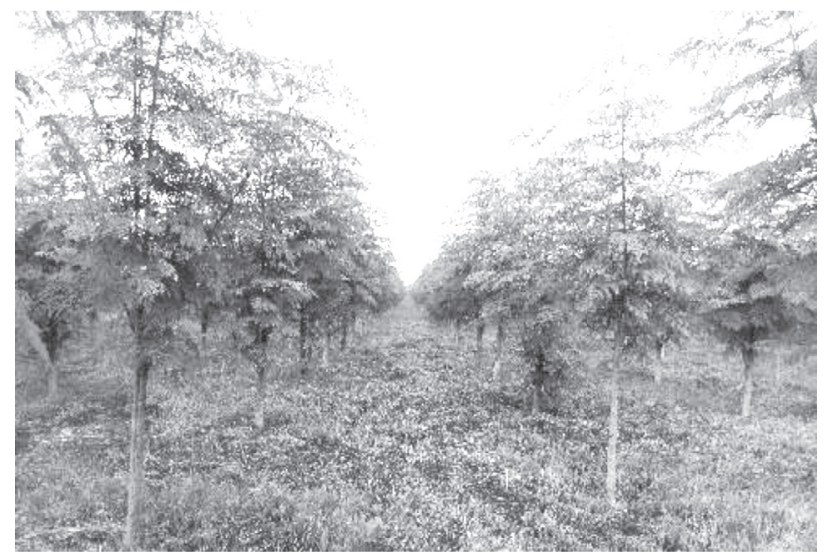

\section{RESUMEN}

Con el propósito de aportar a la identificación con base científica del material reproductivo (especie acriollada y variedad PKM) de Moringa oleifera Lam., con el que trabajan productores nicaragüenses, se llevó a cabo la caracterización morfológica con base en estructuras vegetativas y reproductivas como hojas y configuración de corona o copa y, frutos y semillas. Metodológicamente el trabajo fue desarrollado a través de la observación y medición de las estructuras de interés durante el período de cosecha en instalaciones del Instituto Nicaragüense de Tecnología Agropecuaria (INTA). Los resultados muestran que para todas las variables medidas se encontró diferencias estadísticas, lo que indica diferenciación morfológica marcada entre los materiales reproductivos.

Palabras clave: parámetros morfológicos, diferenciación morfológica.

\section{ABSTRACT}

With the aim to contribute to the scientific identification of Moringa oleifera Lam., reproductive material, was carried out a morphological characterization taking account the vegetative and reproductive structures as leaves and branches configuration and, pods and seeds. The methodology were develop through observation field and measurement of the structures during yield period at Instituto Nicaragüense de Tecnología Agropecuaria (INTA) plantation. The results registered shown values statically different in all variables between the samples, suggesting morphological differentiation.

Key words: Morphological parameters, morphological differentiation.
$M$ oringa oleifera es la especie más conocida del género Moringa. Es un árbol originario del sur del Himalaya, el nordeste de la India, Bangladesh, Afganistán y Pakistán. Se encuentra diseminado en una gran parte del planeta y en América Central (Pérez et al., 2010). En América Central fue introducido cerca de 1920 como planta ornamental y como cercas vivas (Falasca y Bernabé, 2008).

Recientemente ha sido desarrollada en la India una variedad arbustiva y anual de Moringa oleifera conocida como PKM. Esta variedad es de alta producción. Empieza a producir frutos en seis meses y produce más de 300 frutos por planta. PKM ha de ser cortada al final del año y vuelta a plantar de semilla (FAO-OMS, 2005); según el sistema productivo definido.

Las técnicas de caracterización morfológica de especies es un aspecto de interés en la identificación de variantes fenotípicas entre especies y variedades. La taxonomía vegetal plantea el uso de órganos vegetativos o reproductivos para clasificar y diferenciar especies y variedades.

El presente reporte tiene como propósito documentar diferencias morfológicas en dos materiales reproductivos de marango utilizados en Nicaragua, contribuyendo así a la diferenciación en campo por parte de técnicos de las instituciones que promueven este cultivo.
Recibido: 27 de enero del 2017 Aceptado: 19 de abril del 2017

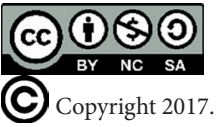

Los artículos de la revista La Calera de la Universidad Nacional Agraria, Nicaragua, se comparten bajo términos de la licencia Creative Commons: Reconocimiento, No Comercial, Compartir Igual. Las autorizaciones adicionales a las aquí delimitadas se pueden obtener en el correo freddy.aleman@ci.una.edu.ni Universidad Nacional Agraria 


\section{METODOLOGÍA}

En la unidad experimental del Centro Nacional de Investigaciones Agropecuarias y biotecnología (CNIA) perteneciente al Instituto Nicaragüense de Tecnología Agropecuaria (INTA) se realizó la caracterización morfológica preliminar de dos materiales reproductivos de Moringa oleifera que constituyen plantaciones mayores a siete años dentro del área experimental.

El material caracterizado corresponde a Moringa oleifera Lam, conocida como especie naturalizada o acriollada en Nicaragua, y Moringa oleifera var. PKM., que es comúnmente conocida como variedad mejorada cuya modificación genética se hizo en el carácter "longitud de fruto" por ser la parte que mayormente se consume entre la población de la India; teniendo así influencia en la producción de semillas.

La caracterización consistió en la cuantificación del número de frutos por árbol para luego obtener un valor medio por tipo de material. Para ello, se seleccionó una muestra de 12 árboles de la especie acriollada; y 16 árboles de la variedad PKM.

Una vez cosechados los frutos, a estos se les midió la longitud $(\mathrm{cm})$ y el diámetro promedio (valor obtenido de dos mediciones o mediciones en cruz del centro de cada fruto, medido en milímetros con vernier digital); así como el número de semillas por fruto (de una muestra de 12 frutos por árbol), y las diferencias entre semillas de cada material.

Para la variable longitud y diámetro de frutos se realizó una categorización en frutos pequeños y frutos grandes; obteniéndose una muestra de 72 frutos pequeños y 72 frutos grandes por material caracterizado.

Un análisis de varianza para cada una de las variables consideradas fue realizado para determinar diferencias estadísticas en los valores medios.

\section{RESULTADOS}

Pérez et al., (2010) describe a la especie acriollada como una especie cuyo habito de crecimiento es arbóreo, al alcanzar altura de hasta nueve metros; mientras otros autores mencionan altura máxima de seis metros (Rolof et al., 2009); por el contrario, para el caso de la variedad mejorada PKM ha sido descrita con un hábito de crecimiento arbustivo con altura de tres a cinco metros.

Una característica diferenciante es la estructura de la corona o copa, que en la especie acriollada va de medianamente regular, en arboles jóvenes, a irregular en arboles maduros; así mismo, la configuración de ramas dentro de la estructura de copa ha sido identificada como una característica diferenciante en las poblaciones de Nicaragua, ya que en arboles jóvenes y sometidos a poda temprana, tienden a presentar copas de forma cónica menos abiertas que el caso de la variedad PKM, donde comúnmente la copa es abierta e irregular.

La forma de ramas en la especie acriollada es observable un desarrollo marcadamente rectilíneo; mientras que en el caso de PKM las ramas son curvilíneas desarrollándose del eje central o fuste principal hacia afuera (figura 1).

Un patrón común entre el material caracterizado es el ángulo de inserción de ramas, que va de 35 a 50 grados; mientras en algunos árboles se observó la presencia de ramas con ángulo de inserción de 90 grados que fue poco común en la muestra; y que posiblemente es un efecto de la poda.

Diferencias morfológicas en la hoja. Los dos parámetros que determinan diferenciación en la hoja de cada material reproductivo son la longitud del peciolo y longitud de la hoja que corresponde a una hoja compuesta. En relación al primer parámetro se registran peciolos de mayor longitud (11.29 $\mathrm{cm})$ en la variedad PKM, siendo estadísticamente diferente $(\mathrm{p}<0.001)$, al determinado para la especie acriollada (8.04 $\mathrm{cm})$; dicha diferenciación es igualmente compartida en el parámetro longitud de hoja, el que fue de $43.78 \mathrm{~cm}$ en PKM y $36.97 \mathrm{~cm}$ en la especie acriollada $(\mathrm{p}<0.001)$ encontrándose diferencias estadística entre el material reproductivo.

Número de frutos por árbol. Los resultados de la comparación del número de frutos por árbol indican una mayor producción para la variedad PKM en comparación a la especie acriollada; registrándose valores inferiores de 55.3 y valores

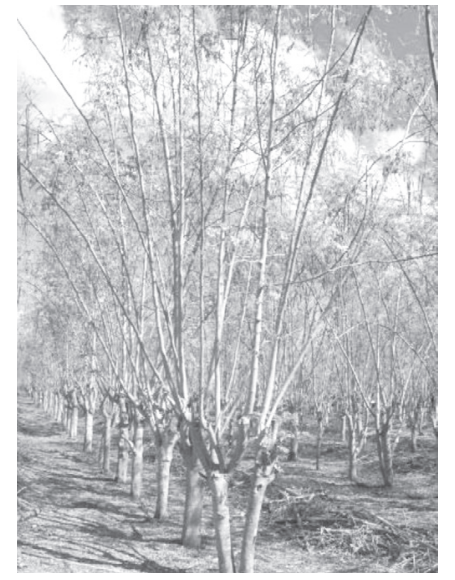

Figura 1. Arboles de material acriollado.

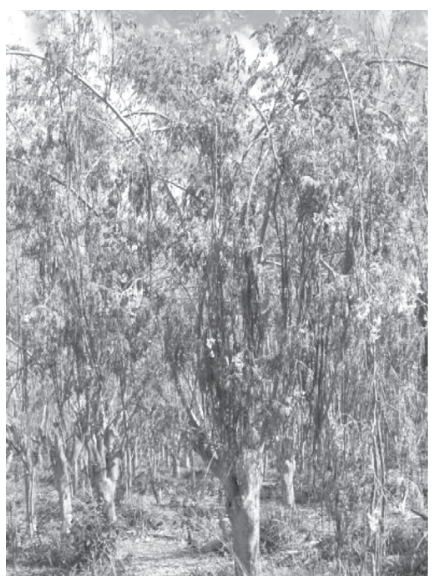

Figura 2. Arboles de variedad mejorada PKM 
superiores de 94.5 frutos por árbol. Diferencias significativas $(\mathrm{p}<0.001)$ se registraron entre los valores medios del número de frutos por árbol para la muestra caracterizada.

Longitud de frutos (silicua) por material caracterizado. De la categorización de los tipos de frutos de la muestra, se realizó una comparación entre tamaño de éstas estructuras reproductivas.

Para el caso de la variable longitud de frutos grandes se determinó una mayor longitud de frutos para la variedad PKM, registrándose valores inferiores de $37.6 \mathrm{~cm}$ y valores superiores $56.2 \mathrm{~cm}$ (figura 3); siendo ambos valores inferiores a los 43.5 y $67 \mathrm{~cm}$ reportados por Pascua, (2014), en plantaciones de la Universidad Nacional Agraria (UNA).
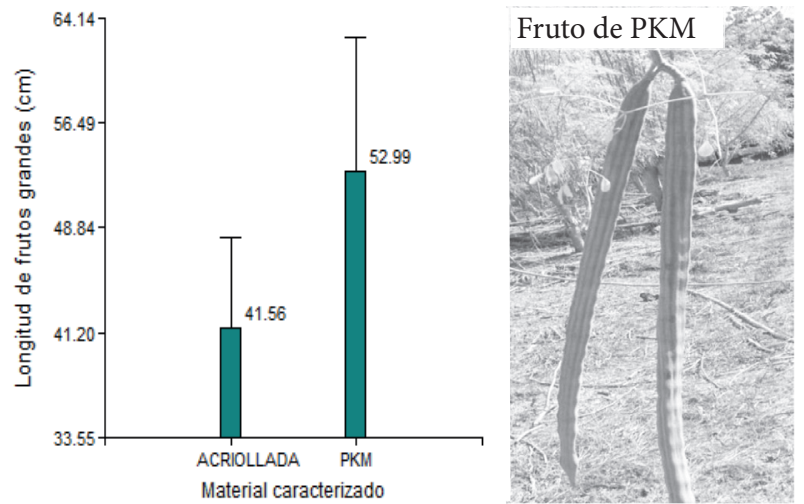

Figura 3. Valores medios de la longitud de frutos grandes por material caracterizado.

Diferencias significativas $(\mathrm{p}<0.001)$ fueron determinadas al comprar los valores medios de longitud de frutos para el tipo de fruto grande. Los resultados de la longitud de frutos grandes para la especie acriollada registrados son similares a los reportados por La Comisión Técnica de Fitomed (2010) y FAO-OMS (2005), siendo de 40 centímetros la longitud máxima registrada.

En cuanto a la variedad PKM, la longitud de frutos grandes es menor a los 70 centímetros según lo reportado por Pérez et al., (2010).

En el caso de los frutos pequeños, se registró la misma tendencia que en el caso de frutos grandes, encontrándose una mayor longitud para el tipo de frutos pequeños en PKM.
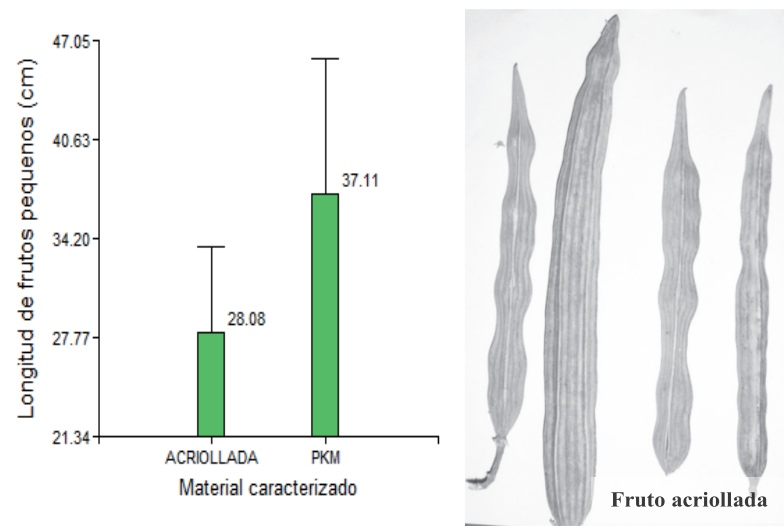

Figura 4. Longitud de frutos pequeños por material caracterizado.
Diferencias significativas fueron determinadas al comprar los valores medios de longitud de frutos para el tipo de frutos pequeños. La longitud de fruto para el tipo de fruto pequeño es superior al mínimo reportado por FAO-OMS, (2005), que fue de 20 centímetros.

Número de semillas por frutos. La cuantificación del número de semillas por frutos muestra similitud entre materiales, encontrándose una ligera superioridad de la variedad mejorada PKM (figura 5), sugiriendo los resultados para el tamaño de muestra analizado similitud en la producción de semillas por material.

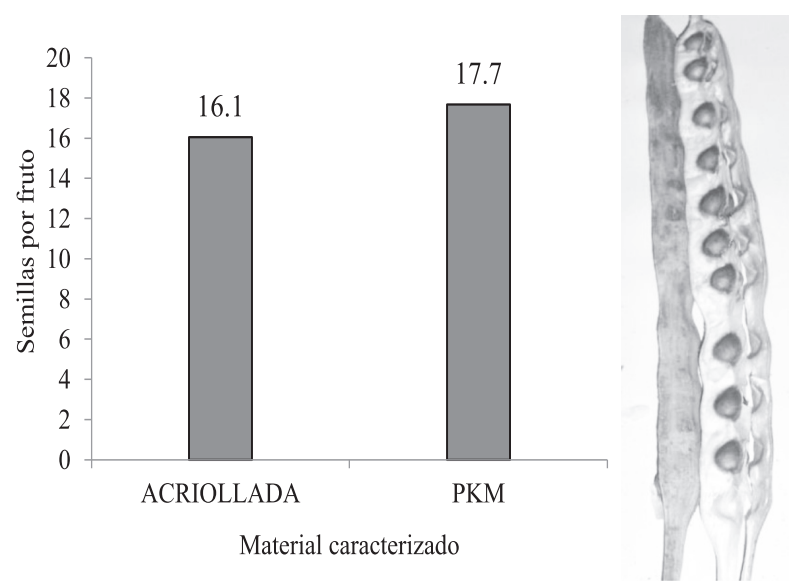

Figura 5. Número de semillas por frutos por material caracterizado.

Diferencias significativas $(\mathrm{p}<0.001)$ fueron determinadas para los valores medios del número de semillas por frutos al comparar entre el material caracterizado; sin embargo, para ambos, el número de semillas por fruto está entre los valores reportados por FAO-OMS, (2005) siendo este entre 12 y 25 semillas; mientras registros del proyecto PROMARANGO de la Universidad Nacional Agraria, reportan valores medios de 21 semillas para el material acriollado y 32 semillas para PKM.

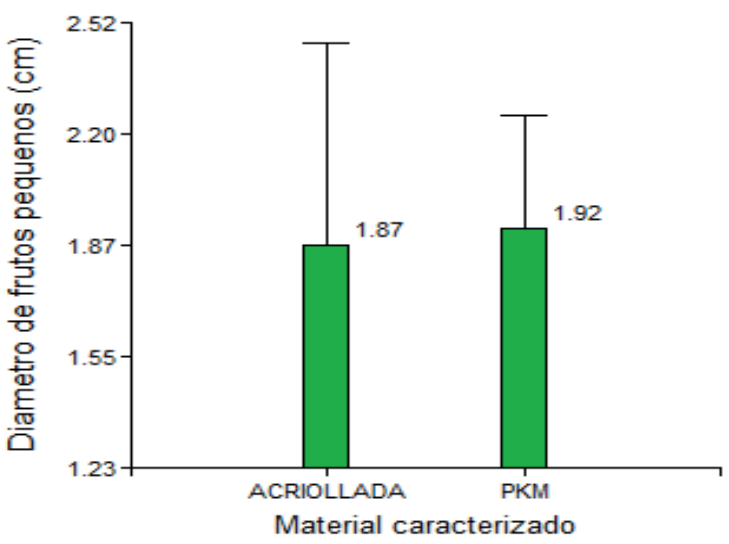

Figura 6. Valores del diámetro de frutos pequeños por tipo de material caracterizado. 
Diámetro por tipo de fruto. Al comparar la variable diámetro por tipo de frutos, no se registraron diferencias estadísticas entre los materiales caracterizados. El mayor diamétricos de los frutos grandes fue para la especie acriollada de 2.09 $\mathrm{cm}$ y 2.04 para la variedad PKM.

En cuanto al diámetro de frutos pequeños (figura 6), los valores indican un mayor diámetro en la variedad PKM, valores que son superiores a 1.31 y 1.50 centímetros reportados por Pascua, (2014).

Diferencias entre semillas de dos materiales reproductivos de marango. Otras variables que permiten diferenciar a las variedades de marango son el tamaño y color de la semi1la. En ambos materiales la semilla presenta formas redondeadas o globulares con tres alas (figuras 7 y 8 ).

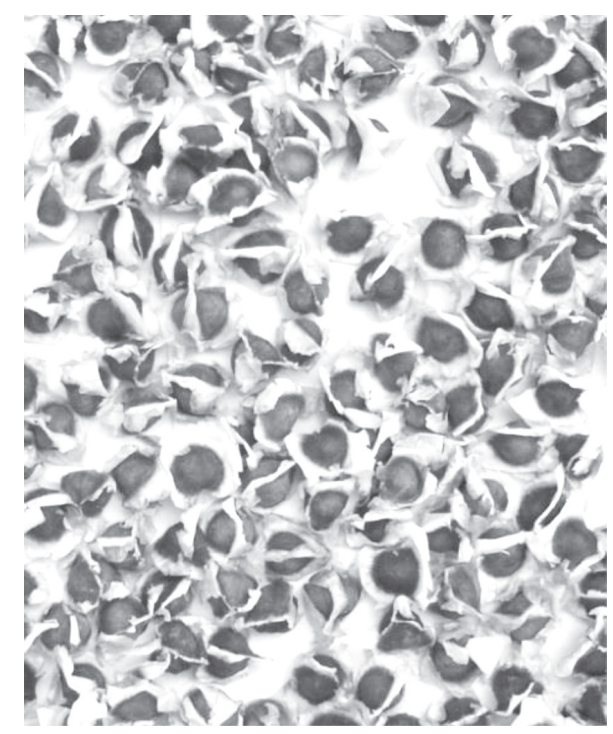

Figura 7. Semillas de la especie acriollada.
La semilla de la especie acriollada presentan una coloración castaño oscuro a pardo oscuro y de tamaño más pequeña que la variedad PKM; ésta última con semilla de color café-castaño. Además, la semilla de la especie acriollada presenta alas de color blanquecina lo que coincide con lo reportado por Foidl et al., (1999), mientras en PKM tiende a un color amarillento; sin embargo, esta característica varia en dependencia de la madurez del fruto y semilla al momento de la cosecha.

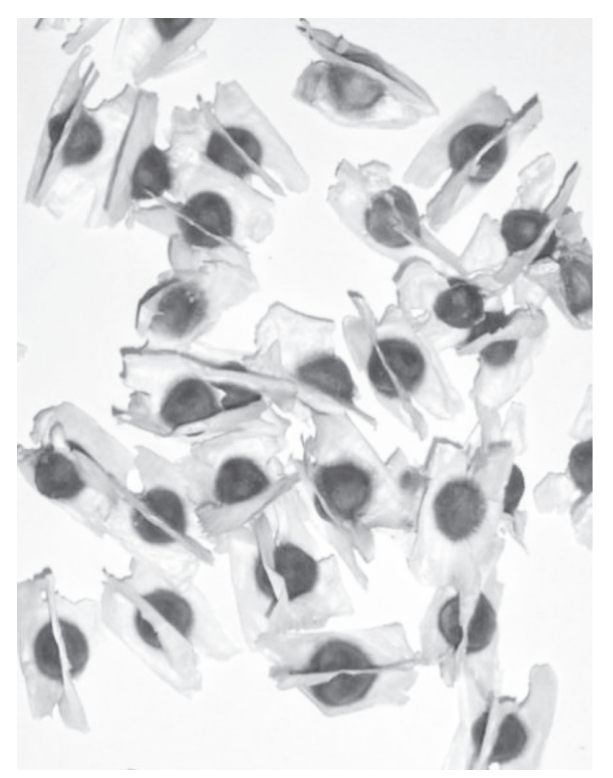

Figura 8. Semillas de la variedad PKM.

\section{CONCLUSIÓN}

Existe diferenciación morfológica entre Moringa oleifera especie acriollada, introducida a inicios del siglo XX, y la variedad mejorada PKM, introducida en los años noventa, lo que permite tomar decisiones sobre el material reproductivo más pertinente con los esquemas de producción definido por cada productor.

\section{REFERENCIAS BIBLIOGRÁFICAS}

Comisión Técnica de Fitomed. 2010. Paraíso francés. (en línea). Consultado 16 abr. De 2013. Disponible en http://www.sld.cu/fito$\mathrm{med} /$ par aiso_frances.htm

Falasca, S; Bernabe, MA. 2008. Potenciales usos y delimitación del área de cultivo de Moringa oleifera en Argentina. Revista vitual REDESMA. Buenos Aires, AR. 16 p.

FAO-OMS (Organización de las Naciones Unidas para la Alimentación y la Agricultura); (Organización Mundial de la Salud). 2005. Normas alimentarias FAO/OMS. Comité del Codex sobre residuos de plaguicidas. Reunión 37. (en línea). Consultado 3 feb. 2010. disponible en http://www.codexali mentarius.net/ download/report/641/al28_24s.pdf.

Foidl, N.; Mayorga, L.; Vásquez, W. 1999. Utilización del marango (Moringa oleifera) como forraje fresco para ganado. Conferencia electrónica de la FAO sobre "Agroforesteria para la producción animal en Latinoamérica". Nicaragua. (en línea). Consultado el 20 de ago. 2014. Disponible en http://www.fao.org/ag/aga/agap/frg/agrofor1/Foidl16.htm

Pascua González, KP. 2014. Ensayo de cuatro procedencias de Marango (Moringa oleifera, Lam) en la Finca Santa rosa, Universidad Nacional Agraria. Tesis Ing. Universidad Nacional Agraria. Managua, NI. 42 p.

Pérez, A; Sánchez, T; Armengol, N; Reyes, F. 2010. Características y potencialidades de Moringa oleifera, Lamark. Una alternativa para la alimentación humana. Pastos y forrajes. Vol. 33. No. 4. Estación Experimental de Pastos y Forrajes "Indio Hatuey". Matanzas, CU.

Rolof, A; Weisgerber, V; Lang, B. 2009. Enzyklopadie der holzgewachseund atlas der Dendrology. 2da Ed. Weinheim. p 1-8. 